\title{
Ordinal pattern statistics for the assessment of heart rate variability
}

\author{
G. Graff ${ }^{1, a}$, B. Graff ${ }^{2}$, A. Kaczkowska ${ }^{1}$, D. Makowiec ${ }^{3}$, J.M. Amigó ${ }^{4}$, J. Piskorski ${ }^{5}$, \\ K. Narkiewicz ${ }^{2}$, and P. Guzik ${ }^{6}$ \\ 1 Faculty of Applied Physics and Mathematics, Gdańsk University of Technology, Poland \\ 2 Hypertension Unit, Department of Hypertension and Diabetology, Medical University of \\ Gdańsk, Poland \\ 3 Institute of Theoretical Physics and Astrophysics, Gdańsk University, Poland \\ ${ }^{4}$ Centro de Investigación Operativa, Universidad Miguel Hernández, Spain \\ 5 Institute of Physics, University of Zielona Góra, Poland \\ 6 Department of Cardiology-Intensive Therapy, University of Medical Sciences in Poznań, \\ Poland
}

Received 27 March 2013 / Received in final form 25 April 2013

Published online 25 June 2013

\begin{abstract}
The recognition of all main features of a healthy heart rhythm (the so-called sinus rhythm) is still one of the biggest challenges in contemporary cardiology. Recently the interesting physiological phenomenon of heart rate asymmetry has been observed. This phenomenon is related to unbalanced contributions of heart rate decelerations and accelerations to heart rate variability. In this paper we apply methods based on the concept of ordinal pattern to the analysis of electrocardiograms (inter-peak intervals) of healthy subjects in the supine position. This way we observe new regularities of the heart rhythm related to the distribution of ordinal patterns of lengths 3 and 4 .
\end{abstract}

\section{Introduction}

Searching for typical features of the sinus rhythms by the analysis of heart rate variability is an important challenge in contemporary physiological and cardiological research. The basic information for this pursuit is provided by the time series of inter-peak intervals (RR intervals) in an electrocardiogram (ECG). The recent development of nonlinear methods in time series analysis makes it possible to reveal new dependencies in heart rate dynamics of healthy subjects. These methods include Poincaré or recurrence plots (Sect. 3), and ordinal patterns (Sect. 3). One of such new observations, made by careful analysis of the Poincaré plot of RR intervals, is heart rate asymmetry (HRA). This is a physiological phenomenon related to the unequal contributions of heart rate decelerations and accelerations to heart rate variability [1]. In order to quantify the deviation from symmetry, some asymmetry indices have been recently defined by means of a two dimensional Poincaré plot: Guzik index [2], Karmakar index [3] and Porta index [4]. Another facet of asymmetry manifests itself

\footnotetext{
${ }^{a}$ e-mail: graff@mif.pg.gda.pl
} 
in terms of deceleration and acceleration runs (i.e., substrings of consecutive RR intervals) of lengths greater than 4 . It turns out that the acceleration runs are longer and more numerous than the deceleration ones [5].

The importance of such kind of rhythm pattern analysis was already pointed out by the Task Force of the European Society of Cardiology and the North American Society of Pacing Electrophysiology [6].

Although there are still few studies on this topic, the drift from asymmetry might be a valuable prognostic indicator of different abnormalities in human physiology. For example, heart rate variability related to heart rate decelerations and accelerations is reduced in patients with type 1 diabetes compared to healthy individuals both in resting 10-min and ambulatory 24-h ECGs [7]. Also, the number of deceleration runs is a powerful parameter in the assessment of post-infarction risk [8].

The aim of this paper is to generalize the approach published in [5], based on the comparison of increasing and decreasing runs, as well as the approach of [3], which divides the runs of length 3 into two clouds. To this end we study all runs of length 3 and 4, together with the distributions of the ordinal patterns defined by the RR intervals. As a result, we obtain an overall picture of the dependencies appearing in a sinus rhythm. The ordinal pattern analysis, which we apply in our study, is a very promising device that originates in the papers of Bandt, Keller and Pompe $[9,10]$. This method has been successfully applied in the analysis of various types of real time series, including biomedical signals derived from electroencephalograms [11-14] and ECGs [15].

This paper is organized as follows. In Sect. 2 we define the indices proposed so far in the literature to quantify the heart rate asymmetry. The instrumental concept of ordinal pattern is introduced then in Sect. 3. Sect. 4 deals with the procedural aspects related both to data acquisition and its numerical processing. The results are presented in the core Sect. 5 . The paper ends with a general discussion and the conclusions.

\section{Asymmetry indices}

Let us consider a finite time series $\left\{x_{n}\right\}_{n=1}^{N}$. The associated planar set $P=$ $\left\{\left(x_{n}, x_{n+1}\right): 1 \leq n \leq N-1\right\}$ is often called a Poincaré plot. We will study the time series generated by heart beats, i.e., $x_{n}=R R_{n}$, where $R R_{n}$ is the $n$th time interval between consecutive heart beats. When we speak of Poincaré plots hereafter, we refer to this particular case. A typical Poincaré plot for a healthy subject is an ellipse. In general, the shape of such a Poincaré plot is an important source of information about the state of the organism [16].

The main diagonal (or the identity line) divides the $\left(R R_{n}, R R_{n+1}\right)$-plane into two disjoint parts, $P_{u}$ and $P_{d}$, comprising the points that are situated over or under that line, respectively. Such a division has a clear physiological interpretation: $R R_{n+1}>$ $R R_{n}$ for $P_{u}$ means that this part corresponds to decelerations of the heart rate; analogously, $P_{d}$ corresponds to acceleration.

\subsection{Porta index}

Porta et al. introduced in [4] an index that measures the asymmetry of the distribution of points in the Poincaré plot with respect to the main diagonal. The index evaluates 
the number of points below the main diagonal with respect to the overall amount of points outside the main diagonal.

$$
\operatorname{Ind}_{P}=\frac{\left|P_{d}\right|}{\left|P_{u}\right|+\left|P_{d}\right|} \%
$$

where for any given set $A$ we denote by $|A|$ the number of elements in $A$.

The Porta index was a useful device to detect temporal asymmetries, measured as a deviation from the value $\operatorname{Ind}_{P}=50 \%$. In particular, it was observed in [17] that Ind $_{P}>50 \%$ and that this inequality is more likely over short temporal scales than over longer ones. Morever, this inequality significantly holds in healthy subjects at rest in supine position, and it is best expressed during a $75^{\circ}$ and $90^{\circ}$ head-up tilt test.

\subsection{Guzik index}

Let us define

$$
S D 1_{\text {up }}^{2}:=\frac{1}{n} \sum_{i}\left(D_{u}^{i}\right)^{2}, S D 1_{\text {down }}^{2}:=\frac{1}{n} \sum_{i}\left(D_{d}^{i}\right)^{2}
$$

where $D_{u}^{i}\left(D_{d}^{i}\right)$ denotes the distance of the $i$ th point in $P_{u}\left(P_{d}\right)$ from the main diagonal.

The main finding of Piskorski and Guzik in [2], called by the authors heart rate asymmetry, was the fact that the Poincaré plot is not symmetrical for healthy patients 30 min ECG in resting. Specifically, there was a higher statistically significant contribution from the upper part of the plot than from the lower part: $S D 1_{\text {up }}>S D 1_{\text {down }}$. This shows a one-directional asymmetry, $P_{u}$ having a higher impact than $P_{d}$.

\subsection{Karmakar index}

Karmakar et. al. considered in [3] segments $P_{i}$ consisting of three consecutive RR intervals, $P_{i}=\left(R R_{i}, R R_{i+1}, R R_{i+2}\right)$, and divided the set of all such triples into the following three groups (called clouds).

- Increasing cloud I:

$$
\begin{gathered}
P_{i} \in I \Longleftrightarrow\left(R R_{i}<R R_{i+1} \text { and } R R_{i+1}<R R_{i+2}\right) \text { or } \\
\left(R R_{i} \geq R R_{i+1} \text { and } R R_{i+1}<R R_{i+2}\right) \text { or } \\
\left(R R_{i}>R R_{i+1} \text { and } R R_{i+1} \leq R R_{i+2}\right) .
\end{gathered}
$$

- Decreasing cloud D:

$$
\begin{gathered}
P_{i} \in D \Longleftrightarrow\left(R R_{i}>R R_{i+1} \text { and } R R_{i+1}>R R_{i+2}\right) \text { or } \\
\left(R R_{i} \leq R R_{i+1} \text { and } R R_{i+1}>R R_{i+2}\right) \text { or } \\
\left(R R_{i}<R R_{i+1} \text { and } R R_{i+1} \geq R R_{i+2}\right) .
\end{gathered}
$$


- Neutral cloud N:

$$
P_{i} \in N \Longleftrightarrow R R_{i}=R R_{i+1}=R R_{i+2} .
$$

The heart rate asymmetry in the paper of Karmakar et al. is interpreted as the lack of symmetry among the distribution of points in the clouds I and D. The Guzik and Porta indices are redefined using the clouds I and D instead of the sets $P_{u}$ and $P_{d}$, respectively. The asymmetry is defined by the new index values as the deviation from $50 \%$ greater than $1 \%$ of the difference between the minimum and maximum index values (within the examined group). The redefinition of indices resulted in a higher prevalence of subjects revealing asymmetry in heart rate variability.

\subsection{Indices based on the comparison of acceleration and deceleration runs}

Another important facet of heart rate asymmetry is revealed when comparing consecutive accelerations and decelerations of the same length. This approach was related to the study of long ECGs. In [5] 24h holter ECG recordings of 87 healthy subjects were analyzed and the differences between acceleration and deceleration runs were demonstrated. The former are longer and also more numerous than the latter, with the exception of runs of lengths 3 and 4. Indeed, the longest acceleration run recorded had length 24, to compare with 19 for the longest deceleration one. Moreover, 1940 acceleration runs with lengths over 10 were recorded, but only 463 decelerations ones in that length range [5].

\section{Methods}

The symbolic representation of time series by means of ordinal patterns is a very convenient method of analysis that can be applied to strings of elements from sets equipped with the concept of order [18]. As we are going to show, the use of ordinal patterns sheds a new light on the heart rate asymmetry and generalizes the approaches described in the previous section.

Given a time series $\left\{x_{t}\right\}_{t=1}^{N}$, consider a sliding window of length $L, x_{n+1}^{n+L}:=$ $x_{n+1}, \ldots, x_{n+L}, 0 \leq n \leq N-L$. By ordering the elements of the window, we get

$$
x_{n+\pi_{1}}<x_{n+\pi_{2}}<\cdots<x_{n+\pi_{L}}
$$

which defines the ordinal pattern of $x_{n+1}^{n+L}$ as the permutation $\pi=\left[\pi_{1}, \pi_{2}, \ldots, \pi_{L}\right]$ of $\{1,2, \ldots, L\}$. For example, if $L=5$ and $x_{n+1}=3, x_{n+2}=-7, x_{n+3}=0, x_{n+4}=2.5$, $x_{n+5}=10.1$, then the ordinal pattern of this window is given by the permutation $\pi=[2,3,4,1,5]$. In case that two values in a window coincide, say, $x_{i}=x_{j}$, then, following [18], we set $x_{i}<x_{j}$ if $i<j$. Figure 1 depicts all six ordinal patterns of length 3. The methods based on ordinal patterns have some practical advantages, like simplicity and computational efficiency [18]. The pattern length $L$ should be so chosen that $N \gg L$ !, to allow every possible ordinal pattern of length $L$ to appear in a series of length $N$.

The impact of consecutive RR intervals with equal duration is very small for high sampling frequency. This being the case, we can interpret the asymmetry indices in terms of ordinal patterns as follows. The asymmetry measured by the Porta index can be perceived as an unequal distribution of ordinal patterns of length 2 , the pattern $[1,2]$ corresponding to the points in $P_{u}$ and $[2,1]$ to the points in $P_{d}$. Similarly if we ignore the influence of equal RR intervals, then the Karmakar index is related to the partition of all triples of $\mathrm{RR}$ intervals into two clouds: $\mathrm{D}$, consisting of the ordinal 


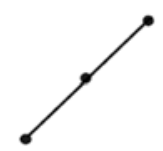

$[2,3,1]$

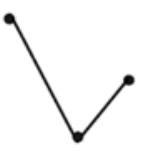

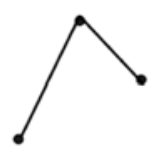

$[3,1,2]$

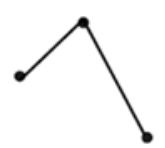

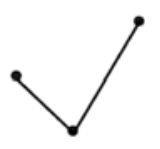

$[3,2,1]$

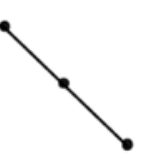

Fig. 1. The six ordinal patterns of length 6 .

patterns $[1,2,3],[2,1,3],[2,3,1]$, and I, consisting of the patterns $[3,2,1],[3,1,2]$, $[1,3,2]$.

Furthermore, the acceleration and deceleration indices of length $k$ can be perceived as the comparison of the number of patterns $[k, k-1, \ldots, 1]$ versus $[1,2, \ldots, k]$. Therefore, these two monotonic patterns will be aptly called acceleration and deceleration patterns, respectively.

In 2012 Parlitz et al. [15] successfully used ordinal pattern analysis (with some time lags) to differentiate patients suffering from congestive heart failure from healthy subjects, showing the strong diagnostic value of this method in cardiology.

Along these lines, we provide below the overall description of the distribution of ordinal patterns of length 3 and 4 in healthy patients, which might be very useful as a reference tool for identifying pathological states.

In particular, to describe the symmetries within groups of ordinal patterns of length 4 , we define the following three self-transformations of the set of all permutations of length 4 :

$$
\begin{aligned}
& R([x, y, z, t])=[t, z, y, x], \\
& P([x, y, z, t])=[z, t, x, y], \\
& S([x, y, z, t])=[y, x, t, z] .
\end{aligned}
$$

Notice that the inverse of each of the above transformation coincide with the transformation itself (i.e., they are involutions).

\section{Procedures}

\section{Subjects}

The group consisted of 97 healthy volunteers (19-40 years old). The study complies with the Declaration of Helsinki; an informed consent was obtained from each patient. The project was approved by the University Bioethics Committee. 
Table 1. Results for $L=3$; $\operatorname{card}_{a v}$ denotes the average frequency of patterns for cardiac data from 94 patients, CI - confidence interval for $95 \%$ mean value.

\begin{tabular}{|c|cc|}
\hline Pattern & card $_{a v}$ & CI \\
\hline \hline 123 & 25,45 & 0,94 \\
321 & 24,25 & 1,06 \\
\hline 231 & 13,36 & 0,49 \\
312 & 13,16 & 0,49 \\
\hline 132 & 11,99 & 0,49 \\
213 & 11,79 & 0,45 \\
\hline
\end{tabular}

\section{Physiological measurements}

For each patient a 30 min ECG was recorded (sampling frequency of $1600 \mathrm{~Hz}$ ) while resting in a supine position. In 3 subjects the number of artifacts and ectopic beats exceeded $5 \%$ of all RR intervals, so they were excluded from further analysis. For the rest of subjects analyzes were performed from the non-edited data.

We calculated the proportions of all possible ordinal patterns in windows of 3 and 4 consecutive RR intervals, with special focus on the acceleration and deceleration patterns.

\section{Comparison with artificial data}

In order to systematically exclude simple explanations of time series observations such as, for example, in case of independent random numbers or Gaussian linear processes, it is advised to compare the results with those obtained by the same analysis but performed on specially preprocessed data [19]. Therefore we also analyzed properties of artificial signals of the two following types.

The signals of the first type are the same cardiac signals but with values of RR shuffled randomly. We call these signals shuffles. With this data we could test the hypothesis that our signals consist of independent draws from a fixed probability distribution. If there are significant differences between the data and the shuffles, then we can reject the hypothesis of independence.

The second type of artificial signals were created under the constrain that the new series had the same Fourier amplitudes as the original data but their Fourier phases were random. We refer to them as surrogates. Due to the Wiener-Khinchin theorem, the Fourier amplitudes relate to the autocorrelation function. Consequently, the sample randomized in this way has the same second order properties (the variance and the auto-covariance function) as the experimental data but it is otherwise random. With this data we could test the hypothesis whether all structure found in our observations were exhausted by properties of some linear stochastic process.

\section{Results}

The frequency of ordinal patterns of length $L=3$ and $L=4$ were investigated. The results for $L=3$ in the form of averages for 94 patients are presented in Table 1 . Observe that the highest frequencies are achieved for the deceleration and acceleration patterns. Among the other patterns one can distinguish two groups with similar frequencies, the first one comprising the patterns $\{[2,3,1],[3,1,2]\}$, and the second the patterns $\{[1,3,2],[2,1,3]\}$. 


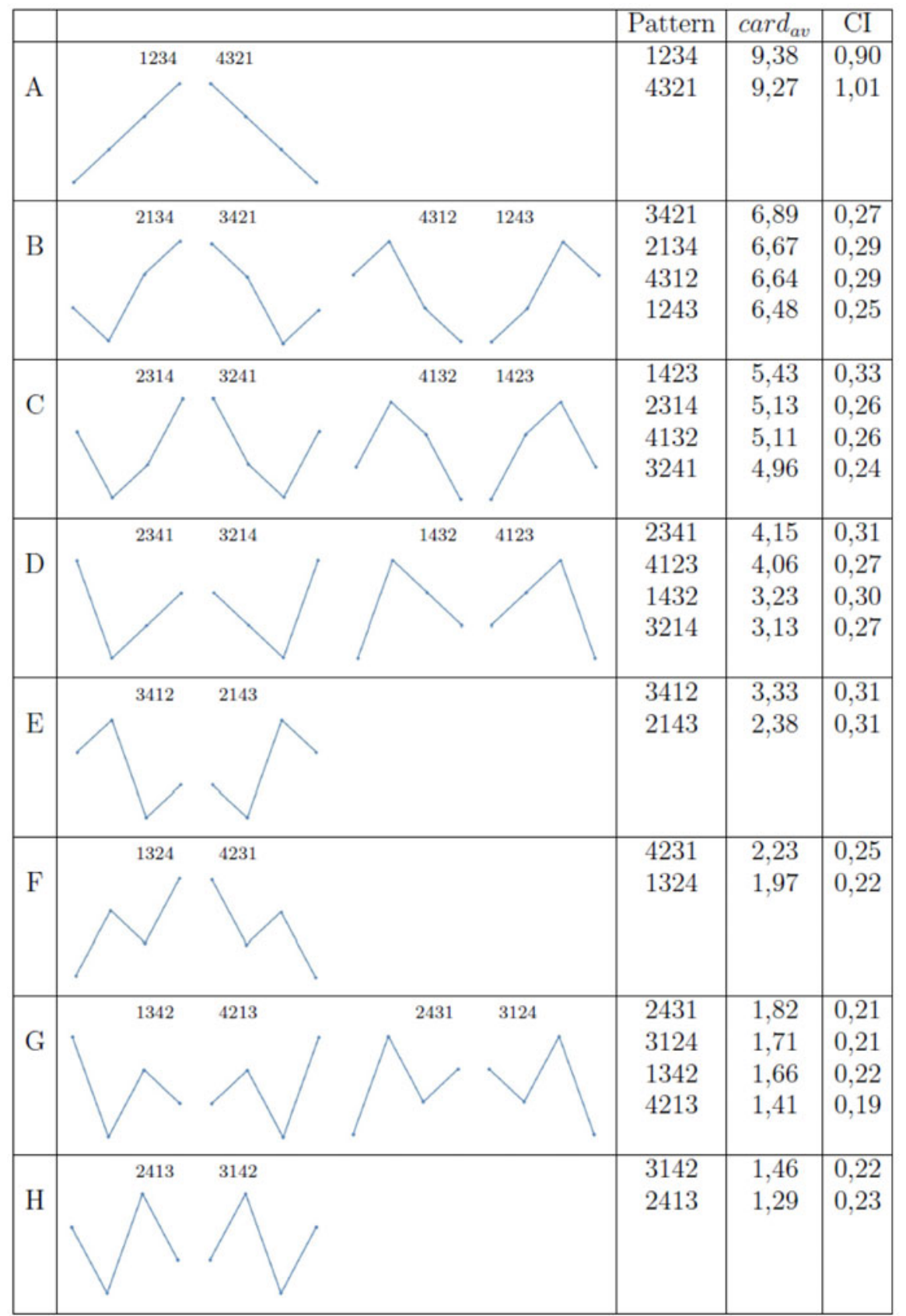

Fig. 2. Patterns of length $L=4$ gathered in groups, $\operatorname{card}_{a v}$ denotes the average frequency of patterns for cardiac data from 94 patients, CI-confidence interval for $95 \%$ mean value.

The interesting phenomenon of an uneven distribution of patterns is clearly visible for $L=4$. The results (see Fig. 2) can be divided in some groups $(A-H)$ so that the frequencies are similar within each group, and the patterns in each group are symmetric.

Each of the groups A,E,F,H consists of one pattern pair invariant under the transformation $R$ (cf. the formula (10)) which could be interpreted as reversibility in time. 
The symmetries in the structure of the groups B and G might be described by the following commutative diagram with the use of the transformations $R$ and $P$ (cf. the formulas (10) and (11)):

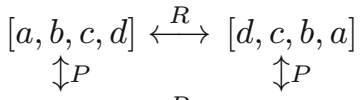

$$
\begin{aligned}
& {[c, d, a, b] \stackrel{R}{\longleftrightarrow}[b, a, d, c] .}
\end{aligned}
$$

Putting anywhere in the diagram (13) any pattern which belongs to B (or G) one can obtain all the remaining patterns in the group B (respectively $G$ ) by a use of transformations appearing in the diagram (13). For example identifying the pattern $[4,3,1,2] \in B$ with $[a, b, c, d]$ we obtain $R([a, b, c, d])=[d, c, b, a]=[2,1,3,4] \in B$, $P([a, b, c, d])=[c, d, a, b]=[1,2,4,3] \in B, P \circ R([a, b, c, d])=[b, a, d, c]=[3,4,2,1] \in B$.

Analogously, the symmetries in $\mathrm{C}$ and $\mathrm{D}$ are illustrated by the diagram:

$$
\begin{array}{cc}
{[a, b, c, d]} & \stackrel{R}{\uparrow_{S}}[d, c, b, a] \\
& \\
\uparrow_{S} & \\
{[b, a, d, c]} & \stackrel{R}{\longleftrightarrow}[c, d, a, b] .
\end{array}
$$

Notice that for $L=4$ (as also for $L=3$ ) the highest frequencies are achieved by the deceleration and acceleration patterns. On the other hand, there are no frequency differences between the patterns $[1,2,3]$ and $[3,2,1]$, as well as between $[1,2,3,4]$ and $[4,3,2,1]$, which is in the agreement with [5].

In Fig. $3(L=3)$ and Fig. $4(L=4)$ we compare the relative frequencies of all length- $L$ ordinal patterns obtained with the cardiac data, shuffles, and surrogates. For the shuffled data, all patterns appear with the same frequency in both cases.

The differences between cardiac data and surrogates appear only in some particular cases. For $L=3$ there are differences between the respective patterns of the form: $[1,2,3],[2,1,3],[1,3,2]$. On the other hand, for $L=4$ the differences are visible mainly within the groups $D \cup E \cup F$, where all the surrogates appear with similar frequencies, unlike the cardiac data.

\section{Discussion and conclusions}

The ordinal pattern-based approach presented in this study provides a more general view on the problem of heart rate asymmetry than the asymmetry indices presented in the literature: Porta index, Guzik index, Karmakar index and acceleration indices.

The main results of our study consist of two observations. Firstly, the pattern distribution is not even, although the ordinal patterns group into some symmetric packages. Secondly, the significant difference between real cardiac data and surrogates unveils a nonlinear structure of the cardiac data, concentrated on some specific patterns. What is more, a random shuffle of the cardiac data equalizes the pattern frequency, which demonstrates that the unequal distribution of patterns is a real physiological phenomenon.

It seems that these findings might be related to the autonomic regulation of heart rate. Symbolic dynamics methods were previously shown to reflect changes of sympathetic and parasympathetic modulation [20]. The heart rate is mainly under the control of the autonomic nervous system. Its two parts have opposite effects: an increased activation of the sympathetic nervous system rises the heart rate, whereas an increased activation of the parasympathetic branch makes the heart rate slower. In normal subjects both the sympathetic and the parasympathetic (vagal) parts of the autonomic nervous system are activated, maintaining the so-called tone. 
$\mathrm{L}=3$ : pattern frequency and $95 \%$ confidence interval

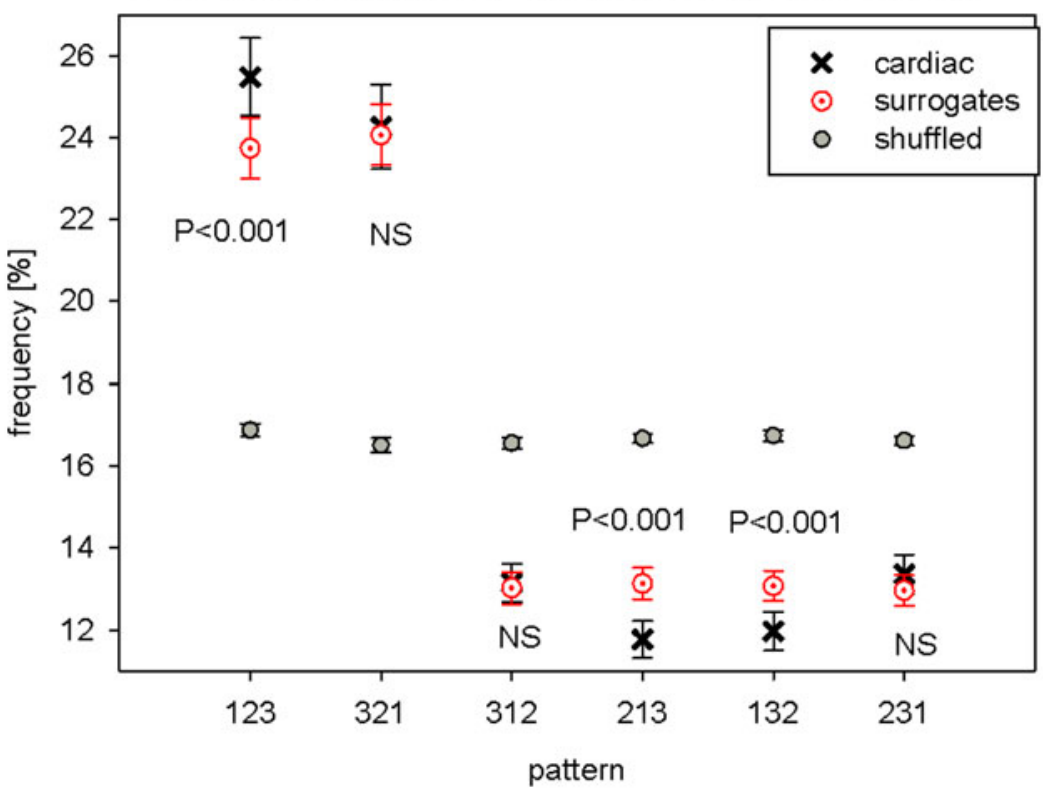

Fig. 3. Frequency diagram of ordinal patterns of length $L=3$ for cardiac data, shuffles and surrogates.

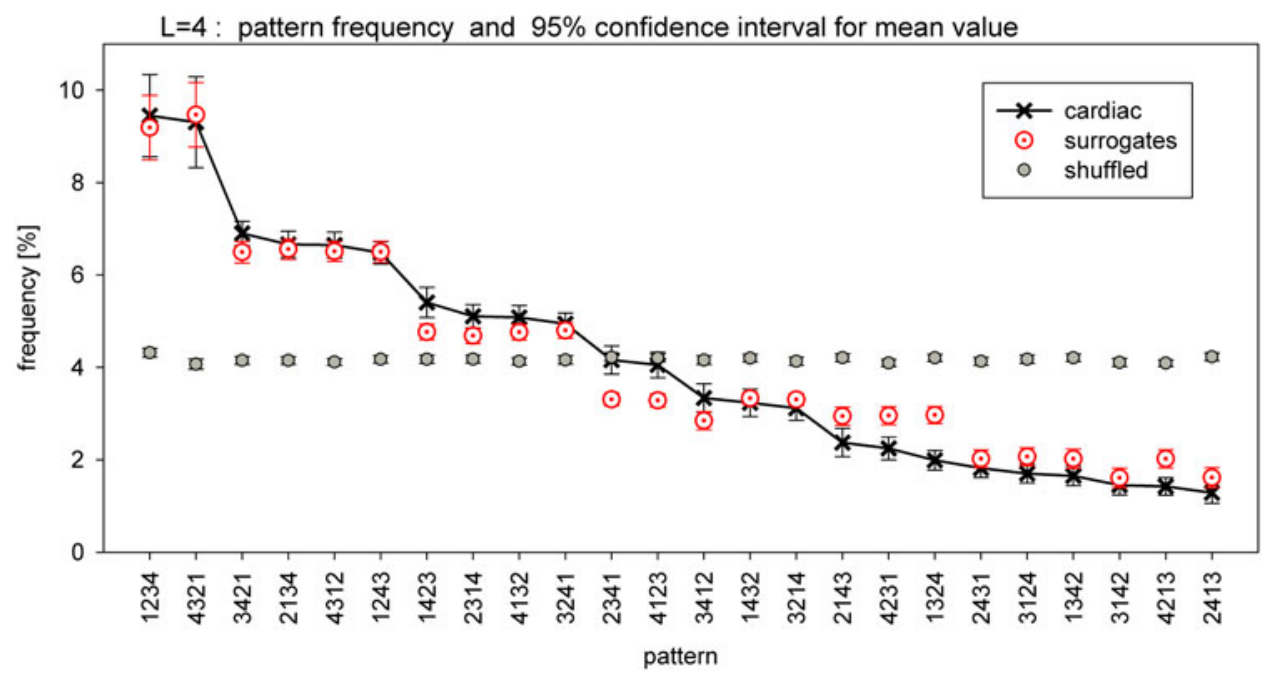

Fig. 4. Frequency diagram of ordinal patterns of length $L=4$ for cardiac data, shuffles and surrogates.

There are some mechanisms that adjust the heart rate depending on the temporary needs of the body using sensors of arterial blood pressure (baroreceptors), level of blood gases (chemoreceptors) and pressure inside the heart (mechanoreceptors). Then the information is transferred to the brain and integrated there, resulting in an appropriate change of the sympathetic and parasympathetic tone. The parasympathetic effect is very fast, almost immediate, while it takes more time to observe the result of a sympathetic activation. Therefore, we hypothesize that in our study, 
"zigzag"-like patterns are related to vagal modulation while patterns with changes in the same direction (acceleration and deceleration runs) are related to sympathetic influences.

The regularities found in the pattern distribution of the sinus rhythm and the generality of the method show that the ordinal pattern-based approach is a promising method in the study of heart rate variability.

This work was supported by the Foundation of Polish Science TEAM/2008-2/5 (B. Graff and K. Narkiewicz) and TEAM program Predicting adverse clinical outcomes in patients with implanted defibrillating devices, co-financed by the European Union within the European Regional Development Fund. (J. Piskorski and P. Guzik). J.M. Amigó was financially supported by the Spanish Ministry of Science and Innovation, Grant MTM2012-31698.

\section{References}

1. P. Guzik, J. Piskorski, T. Krauze, A. Wykretowicz, H. Wysocki, Biomed Tech. 51, 272 (2006)

2. J. Piskorski, P. Guzik, Physiol. Meas. 28287 (2007)

3. C. Karmakar, A. Khandoker, J. Gubbi, M. Palaniswami, Physiol. Meas. 30, 1227 (2009)

4. A. Porta, S. Guzzetti, N. Montano, T. Gnecchi-Ruscone, R. Furlan, A. Malliani, Com. Card. 3377 (2006)

5. J. Piskorski, P. Guzik, Physiol Meas. 32, 1011 (2011)

6. Task Force of the European Society of Cardiology and the American Society of Pacing and Electrophysiology. Heart rate variability: standards of measurement, physiological interpretation, and clinical use, Circulation 93, 1043 (1996)

7. P. Guzik, J. Piskorski, P. Contreras, E. Migliaro, Clin Auton Res. 20, 255 (2010)

8. P. Guzik, J. Piskorski, P. Barthel, A. Bauer, A. Müller, N. Junk, K. Ulm, M. Malik, G. Schmidt, J. Electrocardiol. 45, 70 (2012)

9. C. Bandt, B. Pompe, Phys. Rev. Lett. 88, 174102 (2002)

10. C. Bandt, G. Keller, B. Pompe, Nonlinearity 15, 1595 (2002)

11. J.M. Amigó, S. Zambrano, M.A.F. Sanjuán, Europhys. Lett. 90, 10007 (2010)

12. K. Keller, H. Lauffer, M. Sinn, Chaos Complexity Lett. 2, 247 (2007)

13. K. Keller, M. Sinn. In: Proceedings ICDA 2007, World Scientific 2010, 299

14. G. Ouyang, C. Dang, D. Richards, X. Li, Clin. Neurophysiology 121, 694 (2010)

15. U. Parlitz, S. Berg, S. Luther, A. Schirdewan, J. Kurths, N. Wessel, Comput. Biol. Med. 42, 319 (2012)

16. M. Brennan, M. Palaniswami, P. Kamen, IEEE Trans. Biomed. Eng. 48, 1342 (2001)

17. A. Porta, K.R. Casali, A.G. Casali, T. Gnecchi-Ruscone, E. Tobaldini, N. Montano, S. Lange, D. Geue, D. Cysarz, P. Van Leeuwen, Am. J. Physiol. Regul. Integr. Comp. Physiol. 295, 550 (2008)

18. J.M. Amigó, Permutation Complexity in Dynamical Systems: Ordinal Patterns, Permutation Entropy and All That (Springer Verlag, Berlin, 2010)

19. T. Schreiber, A. Schmitz, Physica D 142, 346 (2000)

20. A. Porta, E. Tobaldini, S. Guzzetti, R. Furlan, N. Montano, T. Gnecchi-Ruscone, Am. J. Physiol. Heart Circ. Physiol. 293, H702 (2007) 